Iustitia Socialis. Revista Arbitrada de Ciencias Jurídicas.

Año IV. Vol. IV. N6. Enero - Junio 2019

Hecho el depósito de Ley: FA2016000064

ISSN: 2542-3371

FUNDACIÓN KOINONIA (F.K). Santa Ana de Coro, Venezuela

Ana Cristina Chirinos Martínez; Rubén Ulises Perozo Martín

DOI: $\underline{\text { http://dx.doi.org/10.35381/racji.v4i6.291 }}$

\title{
Incidencias de la Tecnología web 2.0 en el contexto de la gobernanza y la gobernabilidad
}

\section{Incidents of web 2.0 Technology in the context of governance and governance}

\author{
Rubén Ulises Perozo Martín \\ rpmartin14@gmail.com \\ Universidad Nacional Experimental Francisco de Miranda \\ Venezuela \\ https://orcid.org/0000-0001-8358-905 \\ Ana Cristina Chirinos Martínez \\ chirinos.anacristina@gmail.com \\ Universidad Nacional Experimental Francisco de Miranda \\ Venezuela \\ https://orcid.org/0000-0002-7237-8665
}

Recibido: 11 de abril del 2019

Aprobado: 29 de mayo del 2019

\section{RESUMEN}

La Web 2.0 se considera como la evolución vislumbrada en Internet, desde las webs tradicionales a las aplicaciones dirigidas a usuarios, cuyo uso ha sido la interacción y redes sociales; que pueden servir contenido introduciendo webs interactivas y visuales. El presente artículo tiene como objetivo analizar los enfoques teóricos referentes a la Web 2.0, contribución en la rendición de cuentas en la gobernanza, gobernabilidad; gobierno abierto, transparencia y la participación ciudadana La metodología consistió en efectuar un recorrido sobre artículos arbitrados e investigaciones científicas sobre el desarrollo de diversas aplicaciones en Internet a partir de fuentes primarias. Concluyéndose que el uso de la Web 2.0 debe apuntar a mejorar las relaciones de los gobiernos con sus ciudadanos, otras dependencias gubernamentales y con la empresa privada, para elevar los niveles de confianza de los ciudadanos en sus gobiernos y eliminar la opacidad en los procesos administrativos y de rendición de cuentas.

Descriptores: tecnología web 2.0; gobernanza; gobernabilidad; administración pública 


\section{ABSTRACT}

Web 2.0 is considered as the evolution envisioned on the Internet, from traditional websites to user-directed applications, whose use has been interaction and social networks; that can serve content by introducing interactive and visual webs. The objective of this article is to analyze the theoretical approaches related to Web 2.0, contribution to accountability in governance, governance; Open Government, Transparency and Citizen Participation The methodology consisted of conducting a tour of refereed articles and scientific research on the development of various Internet applications from primary sources. Concluding that the use of Web 2.0 should aim to improve the relations of governments with their citizens, other government agencies and with private enterprise, to raise the levels of confidence of citizens in their governments and eliminate opacity in administrative processes and accountability.

Descriptors: web 2.0 technology; governance; governability; public administration

\section{INTRODUCCIÓN}

En los últimos años han ocurrido cambios políticos, económicos, sociales y culturales, en América Latina, paralelamente a las grandes innovaciones tecnológicas en el área de la informática y telecomunicaciones, que se están dando a nivel mundial, con lo cual se ha elevado el nivel de conciencia política de la sociedad latinoamericana, concediéndoles a los ciudadanos papel protagónico en la formulación, gestión y seguimiento de las políticas públicas. Con empleo de las Tecnologías de la Información y las Comunicaciones (TIC) para dar apertura a la posibilidad de crear un nuevo espacio virtual en las interrelaciones humanas (Gómez, 2018).

Uno de los rasgos característicos de la sociedad moderna es el aprovechamiento intensivo del conocimiento, cuya generación y apropiación tiene una importancia estratégica. En la actualidad la sociedad del conocimiento involucra un conjunto complejo de procesos que puede ser evaluado por los efectos que produce. Tal vez el más importante, es el conjunto de transformaciones que ha generado en el actual entramado social, producto de las necesidad imperante de las instituciones para modernizarse con el uso de herramientas tecnológicas; como medio idea para lograr un mayor acercamiento con los usuarios (Weffer, 2018).

De acuerdo con Sanz (2000), la sociedad del conocimiento surge como consecuencia de los cambios que inducen en la sociedad una serie de innovaciones tecnológicas 
Iustitia Socialis. Revista Arbitrada de Ciencias Jurídicas.

Año IV. Vol. IV. N6. Enero - Junio 2019

Hecho el depósito de Ley: FA2016000064

ISSN: 2542-3371

FUNDACIÓN KOINONIA (F.K). Santa Ana de Coro, Venezuela

Ana Cristina Chirinos Martínez; Rubén Ulises Perozo Martín

desarrolladas en tres sectores convergentes: la informática, las telecomunicaciones, Internet y los medios de comunicación. De igual manera, estas innovaciones tecnológicas han impactado los modelos gerenciales públicos, entre otras cosas, debido a la cobertura espacial, rompimiento de las fronteras físicas, inmediatez de las comunicaciones, interacción entre gobierno-ciudadano.

En ese contexto, la gobernabilidad, es entendida como "un estado de equilibrio dinámico entre el nivel de las demandas societales y la capacidad del sistema político (estado/gobierno) para responderlas de manera legítima y eficaz" (Camou 2001). Que no escapa de los impactos producidos por los avances en las tecnologías de información y comunicación. Así, es importante encontrar una manera de aprovechar los resultados del desarrollo tecnológico, a fin de fortalecer la relación ciudadanogobierno, que permita el uso intra e intergubernamental, con el fin de mejorar los procesos de la gerencia pública y de la política gubernamental. En este caso, consideramos que debe alinearse el uso de las herramientas de tecnologías de información y comunicación, incluyendo los microblogging, con los objetivos estratégicos de la organización para obtener resultados efectivos.

En la primera década del actual milenio se profundiza en la gerencia modelos administrativos como: gerencia de nuevo servicio público, la nueva gerencia pública, adicionándose al modelo burocrático. Basados en caos, complejidad así como también el capital intelectual; los cuales enfatizan la competitividad donde se estudia a la organización de manera transdisciplinaria. Se analizan estos modelos en el contexto de la sociedad del conocimiento, con un amplio uso de las Tecnologías de Información y Comunicación (TIC's), como herramienta para mejorar la gerencia pública o gubernamental.

Posteriormente, caracterizamos el modelo de gobierno electrónico de Venezuela, a fin de determinar si se está usando las aplicaciones Web 2.0, como una herramienta para mejorar su rendición de cuentas para satisfacer las necesidades de los ciudadanos, proporcionando servicios más personalizados del sector público. Finalmente, analizamos el impacto de la tecnología de la cadena de bloques en los atributos de 
Iustitia Socialis. Revista Arbitrada de Ciencias Jurídicas.

Año IV. Vol. IV. N6. Enero - Junio 2019

Hecho el depósito de Ley: FA2016000064

ISSN: 2542-3371

FUNDACIÓN KOINONIA (F.K). Santa Ana de Coro, Venezuela

Ana Cristina Chirinos Martínez; Rubén Ulises Perozo Martín

confiabilidad, integridad, transparencia, auditabilidad $\mathrm{y} / \mathrm{o}$ accesibilidad de la gobernabilidad.

Lo anterior surge de la revisión de los enfoques teóricos referentes a los patrones gerenciales, amalgamado en el razonamiento hipotético deductivo, al realizar un recorrido sobre artículos arbitrados e investigaciones publicadas, sobre los impactos que ha tenido el desarrollo de las tecnologías informáticas, en sus dimensiones de tecnología de comunicaciones y desarrollo de aplicaciones, en la gobernabilidad y gobernanza, con especial énfasis en la implementación del gobierno electrónico y en el uso de las herramientas Web 2.0, en el modelo de gobierno abierto. De allí, se configuran lineamientos teóricos que contribuyan a mejorar la rendición de cuentas y lograr satisfacer las necesidades de los ciudadanos. Al proporcionar servicios más personalizados del sector público para dar respuestas a las demandas actuales de las organizaciones; entendiendo el comportamiento organizacional y la necesidad del cambio planificado.

\section{METODOLOGÍA}

Se presenta una investigación analítica y documental, cuya información se obtuvo partiendo del análisis de artículos e investigaciones previas que recogen las últimas innovaciones plasmadas en material bibliográfico como son: libros, artículos científicos y consultas realizadas en línea. Se sustenta a partir de la recopilación, revisión, y análisis de referencias en inglés y español de libros, artículos indexados en bases de datos: SciELO, Springer Journals, Science Direct, Dialnet y Google Académico.

\section{DESARROLLO}

\section{Gobierno Electrónico}

Las últimas dos décadas, se han caracterizado por la aceleración del desarrollo científico y tecnológico, sobre todo en el área de la informática y telecomunicaciones, que han reconfigurado los modelos de relación en las sectores de la sociedad, impulsando el cambio constante en las organizaciones y funciones sociales. Aun 
cuando la era del internet, nace en la década de los 60, fue en los años 90 que tuvo mayor poder de penetración en la sociedad, debido fundamentalmente por la facilidad de acceso y difusión de las tecnologías de información y comunicación. En los inicios del siglo XXI, con el nacimiento de las redes sociales, se inicia la web 2.0, convirtiendo al internet en una gran aldea global.

A tenor de Castells (2001)a, "El Internet se está convirtiendo en un medio esencial de comunicación y organización en todos los ámbitos de la actividad, es obvio que los movimientos sociales y los agentes políticos lo utilizan y lo utilizarán cada vez más, transformándolo en una herramienta privilegiada para actuar, informar, reclutar, organizar, dominar y contradominar". En el contexto de la gobernabilidad y gobernanza, el internet permite una relación bidireccional, entre ciudadanos y gobierno; a partir de la cual se puede lograr transparencia, planificación coordinada, flujo de información; lo que contribuye por el lado de los ciudadanos a elevar el nivel de identificación con su gobierno, y por el gobierno construir una imagen ante ellos.

El gobierno electrónico, nace en las décadas de los noventa, con el impulso que adquirió las tecnologías de información y comunicación, asociando su concepto al uso de estas tecnologías a la gestión pública, con el fin de facilitar y mejorar la participación de los ciudadanos en el debate público y en la formulación de la política general o de las políticas públicas sectoriales, entre otros medios, a través de consultas participativas a los ciudadanos (González y Pulgar, 2013), democratizando de esta manera las acciones del gobierno.

Desde los inicios del gobierno electrónico, han emergido diferentes conceptos al respecto, por tanto es menester, realizar un análisis de algunas de ellas. El cuadro 1, muestra algunas definiciones de gobierno electrónico. 
Iustitia Socialis. Revista Arbitrada de Ciencias Jurídicas.

Año IV. Vol. IV. N6. Enero - Junio 2019

Hecho el depósito de Ley: FA2016000064

ISSN: 2542-3371

FUNDACIÓN KOINONIA (F.K). Santa Ana de Coro, Venezuela

Ana Cristina Chirinos Martínez; Rubén Ulises Perozo Martín

\begin{tabular}{|c|c|}
\hline Autor & Gobierno Electrónico \\
\hline EzGov (2000) & $\begin{array}{l}\text { "El gobierno electrónico es una manera para que los gobiernos utilicen } \\
\text { las nuevas tecnologías para proporcionar personas con un acceso más } \\
\text { cómodo a la información y servicios del gobierno, a mejorar la calidad de } \\
\text { los servicios y para proporcionar mayores oportunidades a participar en } \\
\text { nuestras instituciones y procesos democráticos" }\end{array}$ \\
\hline Hirst y Nortón,(1998) & $\begin{array}{l}\text { Permitir a los gobiernos a ser más transparentes para los ciudadanos y } \\
\text { las empresas que dan acceso a más de la información generada por el } \\
\text { gobierno; facilitar los cambios fundamentales en las relaciones entre el } \\
\text { ciudadano y el estado, y entre los estados-nación, con implicaciones } \\
\text { para el proceso democrático y estructuras de gobierno. }\end{array}$ \\
\hline Fergunson (1999) & $\begin{array}{l}\text { "Gobierno electrónico implica nuevos estilos de liderazgos, nuevas } \\
\text { formas de debatir y decidir la política y la inversión, nuevas formas de } \\
\text { acceder a la educación, las nuevas formas de debatir y decidir la política } \\
\text { y la inversión, nuevas formas de acceder a la educación, las nuevas } \\
\text { formas de escuchar a los ciudadanos y las nuevas formas de } \\
\text { organización y entrega de información y servicios" }\end{array}$ \\
\hline $\begin{array}{l}\text { Institute of Technology } \\
\text { Assessment, ITA } \\
\text { (1999) }\end{array}$ & $\begin{array}{l}\text { "Gobierno electrónico [...] describe el uso de la nueva información y la } \\
\text { comunicación tecnologías (TIC) para apoyar el funcionamiento de los } \\
\text { gobiernos y el público; administraciones. Por lo general hay tres } \\
\text { principales efector esperados: Mejor y más eficiente los servicios a las } \\
\text { empresas y a los ciudadanos, una mayor eficiencia y transparencia a los } \\
\text { servicios, a las empresas y a los ciudadanos, una mayor eficiencia y } \\
\text { transparencia de la administración pública y ahorro de costos para el } \\
\text { contribuyente." }\end{array}$ \\
\hline Alberta (2000) & $\begin{array}{l}\text { "E-gobierno [...] no se trata sólo de la prestación de los servicios a través } \\
\text { de internet }[\ldots] \text { Los desafíos de enormes proporciones mucho más en los } \\
\text { próximos años es una revolución en el gobierno en sí, un revolución en el } \\
\text { sentido más amplio de la PALABRA. Un cambio dramático en las formas } \\
\text { que el poder político y social e organiza y utiliza" }\end{array}$ \\
\hline Ezgov (2000) & $\begin{array}{l}\text { "Gobierno electrónico es más que un sitio Web, se conecta un gobierno } \\
\text { con sus grupos de interés en una escala que hasta ahora ha sido } \\
\text { imaginable, es el aprovechamiento de internet para simplificar gobierno. }\end{array}$ \\
\hline Pardo (2000) & $\begin{array}{l}\text { "Las iniciativas de gobierno digital están destinados los esfuerzos de } \\
\text { cambio complejos para utilizar las nuevas y las tecnologías emergentes } \\
\text { para apoyar una transformación en la operación y efectividad del } \\
\text { gobierno" }\end{array}$ \\
\hline
\end{tabular}

Fuente: Los investigadores. A partir de Åke Grönlund (2002) 
En términos de lo mostrado en la figura 1, observamos que estas definiciones recogen diferentes perspectivas, sobre gobierno electrónico, colocando énfasis en la relación de ciudadano-gobierno, eficiencia, calidad de servicios, transparencia. Desde la perspectiva de Ferguson (1999), se perfila el gobierno electrónico como un medio, colocando mayor relieve en la cualidad de liderazgo, sin el cual no ocurrirán los cambios esperados. En tal sentido, esta percepción la consideramos acertada, puesto que la inclusión de las herramientas de las TIC's en la Gerencia pública, solo tendrá los efectos deseados, si es acompañada con cambios de actitud y de aptitud de los responsables gubernamentales, añadidos a los de los procesos gerenciales.

Otras definiciones, colocan el punto focal del gobierno electrónico, en la prestación de servicios, tal es el caso del Institute of Technology Assessment (ITA) (1999), además de plantear que a través de la eficiencia se logra un ahorro para los contribuyentes. Esta definición está unida a los nuevos modelos de gerencia pública. En contraste, de las definiciones anteriores, surge la visión emancipadora de Alberta (2000), en la cual infiere que a través del gobierno electrónico, se constituye el espacio ideal para propiciar cambios en el modelo estructural del gobierno, cediéndoles mayor participación a los ciudadanos, es quizás debido a ello, que Castells (2001)a, utiliza los términos de dominación y contra dominación.

Asimismo, es importante destacar que la bibliografía base para el análisis de las diferentes definiciones, data antes del nacimiento de la Web 2.0, año 2004; la cual se ha convertido en una herramienta de comunicación bidireccional, ideal en el de los espacios colaborativos y participativos, y de gran soporte a los modelos innovadores en Gerencia publica: Nueva gerencia publica y el gobierno abierto. La figura 1 muestra tres visiones diferentes sobre el gobierno electrónico en la República Bolivariana de Venezuela. 
Figura 1. Visiones del gobierno electrónico en la República Bolivariana de Venezuela

"El gobierno electrónico en Venezuela significa el aprovechamiento de las tecnologías de información y comunicación para aumentar la inclusión de los amplios sectores que han estado al margen de la acción social del Estado. Las tecnologías de información no sólo pueden propiciar la transformación del estado maximizando las eficiencias de la administración pública, también son un mecanismo para aumentar la transparencia y garantizar la seguridad de la nación" (Gallegos, 2005).

"El gobierno electrónico no es la tecnificación de la burocracia existente, sino que tiene que ver con la inserción de la creatividad y la innovación en la administración pública, para crear procedimientos asociados a viejos y nuevos servicios, que resuelvan y simplifiquen la vida del ciudadano. "(Berrizbeitia, 2005)

"El Gobierno Electrónico es un medio para que el Estado dé respuestas oportunas a las nuevas exigencias de los ciudadanos, reduzca la burocracia, la duplicación de esfuerzos y los retrasos innecesarios" (Mendoza, 2005)

Fuente: Carballo, et al (2006)

Las categorías que emergen al revisar estas visiones son: Inclusión, transformación, eficiencia, transparencia, seguridad, innovación, servicios y simplificación. Consideramos que al igual que en otras perspectivas no se especifica los mecanismos para incrementar la transparencia y la garantía de la seguridad de la Nación, aunado a ello, no se da valor preponderante a la interactividad ciudadano-gobierno, lo cual es un valor agregado que se logra con la implementación de las herramientas de la web 2.0, en el contexto del gobierno electrónico.

En resumen, el gobierno electrónico se define como los procesos de reestructuración para lograr una mayor eficiencia, mejor servicio y una participación más democrática de los ciudadanos, soportadas en el uso de las tecnologías informáticas emergentes. La transparencia y el empoderamiento, son dos elementos fundamentales en los cuales se debe profundizar en la instrumentación del gobierno electrónico. En cuanto a la transparencia, se trata además de los procesos vinculados al modelo gerencial, de la 
Iustitia Socialis. Revista Arbitrada de Ciencias Jurídicas.

Año IV. Vol. IV. N6. Enero - Junio 2019

Hecho el depósito de Ley: FA2016000064

ISSN: 2542-3371

FUNDACIÓN KOINONIA (F.K). Santa Ana de Coro, Venezuela

Ana Cristina Chirinos Martínez; Rubén Ulises Perozo Martín

gestión de los recursos económicos, al respecto Aguilar (2007), citando el informe del banco mundial, al referirse al fracaso del desarrollo de la África negra, afirma que se requiere menos y mejor gobierno, reclamando el cumplimiento de las leyes, la lucha contra la corrupción, la rendición de cuentas, la transparencia en las transacciones y el aseguramiento de la capacidad de los gobiernos para gestionar los recursos económicos.

Desde nuestra postura, consideramos que la connotación de esa cualidad no se logra a través del gobierno electrónico, si bien es cierto, que se puede instrumentar un mecanismo de información de las transacciones presupuestarias financieras de la administración pública, no lo es menos, que esta información adolece de confiabilidad, la cual se alcanza con la conjunción de atributos como la integridad, inmutabilidad, no repudio y disponibilidad; por ello es necesario incluir la cadena de bloques en las tecnologías que involucran al gobierno electrónico.

Por otra parte, el empoderamiento debe ser el fin último de la implementación del gobierno electrónico, de manera de lograr una mayor participación de los individuos y de las comunidades, para impulsar cambios en beneficios de los ciudadanos. Para que se logre el empoderamiento, deben existir políticas y acciones gubernamentales para asegurar el acceso al servicio del internet, la disminución de la brecha digital y un conjunto de leyes que normen y regulen el uso del internet como herramienta gubernamental, así como el uso de estándares para el desarrollo de sitios webs vinculados con el gobierno electrónico, a fin de disminuir la curva de aprendizaje de los ciudadanos al utilizar los diferentes portales vinculados al gobierno electrónico.

De igual manera, podemos afirmar que la perspectiva del modelo del gobierno electrónico, ha evolucionado a la par de los avances del internet, tal como expone Deloite (2008), al describir las características del info gobierno 1.0 y 2.0, haciendo clara vinculación con los cambios de la internet de la 1.0 a 2.0. El info gobierno 1.0, se caracteriza además, de lo descrito por Deloitte (2008), por la baja o nula participación de los ciudadanos en la construcción de las políticas públicas, y apunta a la automatización de los servicios de la administración pública. Los principales aspectos 
diferenciales del infogobierno 2.0, son: La horizontalidad de la gerencia, dinamicidad de la gestión, colaboración, dialogo multimodal entre los actores (gobierno-ciudadanosempresa privada) y transparencia

Cuadro 2: Diferencias entre el info-gobierno 1.0 e info-gobierno 2.0

\begin{tabular}{|c|c|c|}
\hline Dimensión & 1.0 Gobierno & 2.0 Gobierno \\
\hline Modelo operativo & $\begin{array}{l}\text { *Jerárquico } \\
\text { '*Rígido }\end{array}$ & $\begin{array}{c}{ }^{*} \text { Conectada } \\
{ }^{*} \text { Colaboración } \\
\text { *Flexible }\end{array}$ \\
\hline $\begin{array}{c}\text { Los nuevos modelos de } \\
\text { servicio }\end{array}$ & $\begin{array}{c}\text { *Una talla única para } \\
\text { todos }\end{array}$ & $\begin{array}{l}\text { *Personalizada,. } \\
\text { *Basada en elección. }\end{array}$ \\
\hline Entrega & $\begin{array}{c}\text { *Monopoly } \\
\text { *Un canal Solo. }\end{array}$ & $\begin{array}{l}\text { *Multicanal. } \\
{ }^{\star} \text { Resultado }\end{array}$ \\
\hline Toma de decisiones & $\begin{array}{l}{ }^{*} \text { Orientadas a los } \\
\text { insumos. } \\
{ }^{*} \text { Cerrado. } \\
{ }^{*} \text { Espectador. }\end{array}$ & $\begin{array}{l}\text { *Impulsada } \\
\text { *transparente } \\
\text { *Participativa }\end{array}$ \\
\hline
\end{tabular}

Fuente: Deloitte (2008).

Por otro lado, implementar un gobierno electrónico implica, entre otras actividades, redefinir, agregar y/o eliminar procesos, definir políticas de calidad y seguridad, analizar los procesos de negocio en cada uno de los servicios públicos, todo esto en vías de lograr la integración e interoperabilidad de estos servicios. Las soluciones tecnológicas que ayudan a esta tarea deben ser capaces de responder de forma óptima a las necesidades de integración derivadas de la existencia de entornos heterogéneos tanto en aplicaciones como en sistemas.

Asimismo, las consecuencias e impactos en la atención a los ciudadanos se expresan en al menos lo siguientes aspectos: los usuarios pueden recibir atención sin restricción horaria, no importando en qué lugar geográfico se encuentren, los usuarios tienen acceso a información pública en forma simple, oportuna, clara y transparente; los usuarios pueden resolver su problemas a través de un contacto único con el Estado, aunque se trate de requerimientos que involucren a más de una institución; los usuarios no están obligados a presentar ningún documento o certificar información que se 
Iustitia Socialis. Revista Arbitrada de Ciencias Jurídicas.

Año IV. Vol. IV. N6. Enero - Junio 2019

Hecho el depósito de Ley: FA2016000064

ISSN: 2542-3371

FUNDACIÓN KOINONIA (F.K). Santa Ana de Coro, Venezuela

Ana Cristina Chirinos Martínez; Rubén Ulises Perozo Martín

encuentre disponible en formato electrónico por alguna institución pública, los usuarios pueden hacer transacciones financieras en forma electrónica, (por ejemplo pagar un servicio de un documento, o recibir el pago por un servicio prestado un organismo público si se es un proveedor del Estado).

Los ciudadanos son libres para consultar sobre información de los actos públicos del Estado que sea de su interés conocer. El Estado transparentará dichos actos dejándolos disponibles electrónicamente. Los ciudadanos tienen derecho a participar y expresar su opinión por medios electrónicos (e-participation). En el caso del gobierno electrónico de Venezuela, tal como lo establece Carballo et al (2006), existen experiencias emblemáticas como son SENIAT (Servicio Nacional Integrado de Administración Tributaria), SAIME (Servicio Administrativo de Identificación Migración y Extranjería.), SNC (Servicio Nacional de Contrataciones) y los procesos del Instituto Venezolano de Seguro Social, las cuales han permitido la gestión de sus procesos respectivos a través de internet. Mención aparte, por su reciente incorporación, está el sistema carnet de la patria, a través del cual se otorga subsidio directo a los ciudadanos, como parte de las políticas públicas del gobierno nacional.

Estos portales gubernamentales poseen poca interactividad, son administrados por un funcionario público, tienen baja frecuencia de actualización, son de carácter informativo. Podemos concluir, que si bien es cierto que acerca los servicios del gobierno al ciudadano, este no tiene una vinculación activa con el gobierno. De acuerdo al informe anual sobre Gobierno Electrónico 2018 de las Naciones Unidas (Organización de Naciones Unidas, 2018), Venezuela tiene un índice de desarrollo de gobierno electrónico (IDGE) de 0,52877. En términos cualitativos es ubicada en el grupo de alto desarrollo de gobierno electrónico, y en términos cuantitativo en la posición 106 de un universo de 193 naciones. En referencia a la zona geográfica, se ubica por debajo del promedio, el cual es de 0,5898 .

En este contexto, a pesar de presentar una mejora de 0,01597 en el IDGE, con respecto al informe 2016, se muestra un retroceso en la ubicación al pasar del lugar 90 al 106, esto se debe a que otros países han logrado mayores mejoras en los elementos 
que componen el índice. El índice, se construye a partir de tres valores normalizados sobre las dimensiones más importantes del gobierno electrónico: El alcance y la calidad de los servicios en línea, el estado de desarrollo de la infraestructura de telecomunicaciones y el capital humano inherente.

Sin embargo, Venezuela, se ubica en un nivel calidad bajo de servicios en línea, de acuerdo a esto, se infiere que las dimensiones infraestructura de telecomunicaciones y capital humano supera el alcance y la calidad de los servicios en línea, lo cual se corrobora al disgregar los valores del índice, en cuyo caso, son 0.4097, 0.4148 y 0.7615, que al ser comparados con los valores promedios de la zona (0.6095 0.4441 $0.7157)$, se concluye que, se deben implementar acciones a fin de elevar y mejorar los servicios en línea del gobierno electrónico venezolano.

\section{Gobierno Abierto}

En los últimos años sociedades democráticas están enfrentando una crisis de legitimidad (Brugue, 2013), debido fundamentalmente a la opacidad de la gestión pública y de la insatisfacción de los ciudadanos en la solución de sus problemas. Con lo cual se produce disensión entre los ciudadanos y sus gobernantes, contribuyendo a elevar los niveles de desconfianzas en las instituciones y en las clases política; produciéndose un rechazo a la clase política de un país y a las instituciones de los sistemas de gobierno.

Además, diversos gobiernos alrededor del mundo, principalmente de América Latina, han sido señalados de corrupción, opacidad en la administración del erario, ineficacia, falta de rendición de cuentas; desviación de su fines, elitismo en la adopción de decisiones; poca celeridad para la resolución de conflictos de los ciudadanos; elementos que se pueden atribuir, bien sea al modelo de gerencia publica, al bajo nivel de capacitación de los funcionarios, o a la ausencia de ética en los gobernantes y los funcionarios. 
Iustitia Socialis. Revista Arbitrada de Ciencias Jurídicas.

Año IV. Vol. IV. N6. Enero - Junio 2019

Hecho el depósito de Ley: FA2016000064

ISSN: $2542-3371$

FUNDACIÓN KOINONIA (F.K). Santa Ana de Coro, Venezuela

Ana Cristina Chirinos Martínez; Rubén Ulises Perozo Martín

Lo anterior, refleja una preocupación por estudios de la calidad de la democracia y el cuestionamiento acerca de las capacidades con que cuentan esos gobiernos. Generándose un amplio rango de opinión y un número significativo de actores importantes, públicos y privados, que abogan por el Gobierno Abierto.

Existen múltiples definiciones de Gobierno Abierto, en algunos casos son más restrictivas que otras. Se subrayan aquellas que se refieren a la apertura de la información en forma de datos abiertos y la participación de ciudadanos por la vía del desarrollo de aplicaciones tecnológicas que son puestas al servicio del público. Otros cuestionan que el Gobierno Abierto es más que el uso de las tecnologías para instituir colaboración, sino que implica una serie de dimensiones de análisis que se han dejado a un lado. Estos aspectos están vinculados con el servicio público y distintos componentes que promueven la calidad de un gobierno y la confianza de los ciudadanos en su gobierno.

Por su parte, Tapscott (2010) menciona que "Un gobierno, abre sus puertas al mundo, co-invoca con todos, fundamentalmente con los ciudadanos; comparte recursos que anteriormente estaban celosamente guardados, y aprovecha el poder de la colaboración masiva, la transparencia en todas sus operaciones, y no se comporta como un departamento o jurisdicción aislada, sino como una estructura nueva, como una organización verdaderamente integrada y que trabaja en red." Por lo cual, es evidente que un gobierno abierto cumpla con todas sus funciones debe estar construido sobre los siguientes principios o elementos fundamentales:

- La transparencia: toda la información y asuntos de competencia gubernamental deben estar puestos a disposición de todos cumpliendo con varios requerimientos necesarios. Es decir, que el derecho de saber pueda respetarse dando a conocer todas y cada una de las informaciones que la sociedad debe saber.

- La participación ciudadana: todo gobierno debe estar accesible al dialogo, donde se promueva una cultura de interés por los asuntos públicos. Abriendo el paso para gobernar con una verdadera democracia. 
Iustitia Socialis. Revista Arbitrada de Ciencias Jurídicas.

Año IV. Vol. IV. N6. Enero - Junio 2019

Hecho el depósito de Ley: FA2016000064

ISSN: 2542-3371

FUNDACIÓN KOINONIA (F.K). Santa Ana de Coro, Venezuela

Ana Cristina Chirinos Martínez; Rubén Ulises Perozo Martín

- La rendición de cuentas: El gobierno y todo organismo vinculado a este le corresponden justificar todos y cada uno de los movimientos que realizaran en pro de la sociedad, implementando así la transparencia.

De esta manera, el gobierno abierto debe entenderse como una plataforma tecnológica institucional que convierta los datos gubernamentales en datos abiertos para permitir su uso, protección y colaboración por parte de los ciudadanos en los procesos de decisión pública, rendición de cuentas y mejoramiento de los servicios públicos. Esto no es más que un conjunto de tecnologías de información interconectadas a través de un sólo lenguaje utilizando todo el potencial de interacción e interconexión que posibilitan las plataformas. No sólo el uso de internet a través de los sitios web, sino de las plataformas sociales tales como Facebook y Twitter (Dijck, 2016) y del uso de tecnología móvil para difundir e interactuar con la información (Lorenzi et al., 2014).

\section{Critica al gobierno abierto}

El gobierno abierto desde su filosofía presume que una vez disponibles los canales, los ciudadanos estarán disponibles inmediatamente a participar y ejercer funciones que se les atribuye y reconoce discursivamente. ¿Es posible vislumbrar este escenario donde los ciudadanos de la sociedad se conglomeren en un espacio virtual? Pues, desde la perspectiva de Oszlak (2012)

En primer lugar, No es concebible la participación de la sociedad civil en el diseño, puesta en marcha y evaluación de las políticas estatales, a menos que esta haya sido empoderada. En segundo lugar, que el empoderamiento implica que el ciudadano conoce sus derechos individuales y los colectivos, la forma en que se puede obtener la garantía de su ejercicio y la capacidad de análisis de la información pertinente, así como su capacidad de agencia, o sea, de ser o hacer aquello que se tiene razones para valorar, y en tercer lugar que aun empoderado, el ciudadano valora la participación política y tiene la voluntad de ejercerla. (p.70)

En función de lo expresado por Oszlak (2012), suponemos que la tecnología provoca el cambio cultural en la figura de la voluntad política, que debería existir entre el Estado 
Iustitia Socialis. Revista Arbitrada de Ciencias Jurídicas.

Año IV. Vol. IV. N6. Enero - Junio 2019

Hecho el depósito de Ley: FA2016000064

ISSN: 2542-3371

FUNDACIÓN KOINONIA (F.K). Santa Ana de Coro, Venezuela

Ana Cristina Chirinos Martínez; Rubén Ulises Perozo Martín

y la sociedad civil. Pues, si al menos desde el Estado la voluntad política se ejerciera en todos los planos ineludibles, como para eliminar u oprimir las distintas desigualdades e intransigencias. Es posible que una acción sistemática y constante del nivel político pudiera llegar a generar los incentivos necesarios para que esa nueva filosofía penetre y se instale con habitualidad en las prácticas ciudadanas. De modo que la cultura reflejada en esas prácticas llegue a modificarse.

Se trata de exhortar a los funcionarios a que atiendan a los ciudadanos, respondan a sus críticas, observaciones interrogantes o planteamientos y admitan que les corresponden rendirles cuenta. Configurar una relación gobierno-ciudadanía a través del uso de la tecnología, que permitiría esa interacción; aun cuando existan barreras para que los funcionarios políticos y los gerentes se muestren dispuestos a funcionar bajo estas nuevas reglas con una gran voluntad política para lograrlo. (Oszlak, 2012). Evidentemente, la labor extraordinaria que tiene el gobierno abierto es garantizar el acceso a la información a todos los ciudadanos. Esto implica reducir la brecha digital entre sus gobernados, lo cual demanda cambios tanto en infraestructura como en el mercado de las telecomunicaciones y los datos. (Armenta et al., 2012).

\section{Contribuciones de la Web 2.0 en el gobierno abierto}

El desarrollo tecnológico, incide indefectiblemente en las formas y normas de relación de la sociedad, Castells (2001) b, concibe el desarrollo del internet como un medio para promover la cooperación y la libertad de información que puede favorecer la innovación en mayor medida que la competencia. En el contexto de la gobernabilidad, el desarrollo de las redes sociales constituye un vehículo ideal para la implementación del paradigma de gobierno abierto.

De esta manera, el nacimiento de la web 2.0, colocó en el escenario un conjunto de herramientas informáticas para impulsar el intercambio de información entre los ciudadanos y su gobierno, bajo las premisas de transparencia, accesibilidad, colaboración y participación; dando fortaleza al paradigma del gobierno abierto. 
Iustitia Socialis. Revista Arbitrada de Ciencias Jurídicas.

Año IV. Vol. IV. N6. Enero - Junio 2019

Hecho el depósito de Ley: FA2016000064

ISSN: $2542-3371$

FUNDACIÓN KOINONIA (F.K). Santa Ana de Coro, Venezuela

Ana Cristina Chirinos Martínez; Rubén Ulises Perozo Martín

Por su parte Reddick y Aikins (2012), la web 2.0 contempla el desarrollo de las redes sociales y un conjunto de aplicaciones y tecnologías que aprovechan la conectividad de Internet para apoyar el trabajo en red de trabajo colaborativo y el contenido, de manera que constituyen una plataforma para aumentar el acceso a la información y servicios del gobierno, además de facilitar la participación ciudadana en las políticas públicas. El uso de las tecnologías de colaboración y herramientas de medios sociales como blogs, Youtube, Facebook, Twitter, Instagram, y muchos más están transformando las interacciones gobierno-gobierno, gobierno-ciudadano, gobierno-sector privado y ciudadanos-ciudadanos, en materia de gestión pública y gobernabilidad democrática. Además de configurar escenarios novedosos en las convocatorias de la protestas ciudadanas y movilización, difusión de las políticas del gobierno, gestión de las campañas políticas y la participación democrática

En este mismo orden de ideas, destacamos que las aplicaciones Web 2.0 están transformando aceleradamente las interacciones entre los ciudadanos, gobierno y empresa privada de una manera nunca antes vista. En un estudio realizado por Kemp S (2018), sobre internet, las redes sociales y los dispositivos móviles utilizados en todo el mundo para el año 2018; reveló un ritmo de crecimiento mensual de un millón de nuevos usuarios de redes sociales a nivel mundial, además de ubicar en un total de tres (3) billones de personas que usan redes sociales, mensualmente, para comunicarse. El porcentaje de ciudadanos de Venezuela que participan en las redes sociales, actividades de la comunidad virtual, y otros sitios de interés especial se ubica en $44 \%$ de la población general. La red social Facebook, es la de mayor uso con catorce (14) millones de usuarios.

Sin embargo, al contabilizar en el portal twven el número de cuentas del microblogging twitter del dominio del gobierno venezolano es bajo, al igual de la red social Facebook, las cuales son usadas para establecer contactos de manera unidireccional con los ciudadanos. En este contexto, tal como se describe en la encuesta utilizada para determinar el índice de participación en el e-Gobierno 2018 (Naciones Unidas 2018), la administración pública venezolana está empezando, aunque lentamente, a hacer uso 
Iustitia Socialis. Revista Arbitrada de Ciencias Jurídicas.

Año IV. Vol. IV. N6. Enero - Junio 2019

Hecho el depósito de Ley: FA2016000064

ISSN: 2542-3371

FUNDACIÓN KOINONIA (F.K). Santa Ana de Coro, Venezuela

Ana Cristina Chirinos Martínez; Rubén Ulises Perozo Martín

de herramientas interactivas para promover el diálogo, recibir comentarios y aportes de los ciudadanos, así como para proporcionar información y servicios en línea. Esta participación electrónica, esta mayormente orientada a proporcionar información, y en una menor medida a responder a las preguntas y a la toma de decisiones.

Debido a ello, es importante implementar una estrategia para aprovechar la Web 2.0 en el gobierno para fortalecer la relación ciudadano-gobierno y que permita su uso intra e intergubernamental, con el fin de mejorar los procesos de gestión pública y de la política. En este orden de ideas, Webb (2012), realizó una investigación a fin de determinar el uso efectivo de los microblogging, en el marco de la gestión de las políticas públicas en las agencias del gobierno de Estados Unidos. De las veintitrés (23) agencias analizadas, el autor descubrió solo dos (02) agencias con una política de medios centrada el microblogging, otras agencias solo las mencionan en su política de medios. Esta realidad, fue detectada a pesar de los memos emitidos por la administración Obama, en los cuales ordenaban usar de forma "inteligente" el internet, específicamente las redes sociales como medios de vinculación con los ciudadanos.

Por otra parte, al analizar los mensajes que se difunden a través de los microblogging, se estableció la ausencia de políticas en la conformación de los mensajes transmitidos, por parte de las agencias, a través de las redes sociales. En consecuencia, para el uso efectivo de la web 2.0 en el gobierno, es necesario el desarrollo de un sistema de gestión de los medios de comunicación social, en el cual se incluya las redes sociales como elemento fundamental para la interacción con los ciudadanos, el sector privado y con los diferentes niveles del gobierno

En este orden de ideas, existen elementos de seguridad y calidad de información que deben ser abordados previo a la inclusión de las redes sociales en la gestión de las políticas públicas, entre los cuales están: Secrecía, disponibilidad, integridad, confidencialidad, protección, confiabilidad, no repudio y trazabilidad. Al respecto Bertot, et al (2012), proponen una lista de políticas generales sobre el uso de redes sociales gubernamentales. Es necesario que consideremos que cualquier política de uso de estas herramientas debe contemplar la especificad de las redes sociales, de manera 
Iustitia Socialis. Revista Arbitrada de Ciencias Jurídicas.

Año IV. Vol. IV. N6. Enero - Junio 2019

Hecho el depósito de Ley: FA2016000064

ISSN: 2542-3371

FUNDACIÓN KOINONIA (F.K). Santa Ana de Coro, Venezuela

Ana Cristina Chirinos Martínez; Rubén Ulises Perozo Martín

que se establezcan políticas individuales para el uso de cada una de ellas, en el sentido que cada herramienta es única y por lo tanto debe ser tratada como tal.

Adicionalmente, dentro de las políticas propuestas por Bertot et al (2012), destaca la de fomentar la transparencia y la rendición de cuentas, sin embargo, como ya se ha expuesto, a través de las redes sociales no se logra esos fines en su totalidad, y se coincide con Olnesa, et al (2017), al plantear que el uso de la tecnologías de la cadena de bloques, propicia la confiabilidad, transparencia, reducción de la corrupción, inmutabilidad, integridad, accesibilidad y seguridad.

Así de este modo, algunos elementos fundamentales del gobierno abierto tales como; colaboración, construcción colectivas de políticas públicas, comunicación y participación en las decisiones pueden ser alcanzadas de manera efectiva, a través de las herramientas informáticas como los microbloggin, blogs, foros, wiki, entre otros; y la transparencia y rendición de cuentas con la tecnología de las cadenas de bloques Para garantizar los principios fundamentales del paradigma gobierno abierto.

Desde un punto de vista social, las administraciones gubernamentales deben asegurar la accesibilidad a las tecnologías de información y comunicación a los ciudadanos, las cuales de acuerdo a Jaeger et al (2012), se resume en servicio universal, equidad en el acceso e instrucción. A fin de reducir la brecha digital en la sociedad a fin de garantizar la implementación el éxito de implementación del paradigma del gobierno abierto en la sociedad.

\section{Consideraciones finales}

A continuación presentamos las conclusiones relacionadas con el análisis de los enfoques teóricos referentes a la Web 2.0, contribución en la rendición de cuentas en la gobernanza, gobernabilidad; gobierno abierto, transparencia y la participación ciudadana.

El desarrollo tecnológico en el área de las telecomunicaciones e informática, ha revolucionado espacio-temporalmente las formas de asociación social, logrando la unión de un conjunto de voluntades que comparten intereses e ideales, en un espacio 
Iustitia Socialis. Revista Arbitrada de Ciencias Jurídicas.

Año IV. Vol. IV. N6. Enero - Junio 2019

Hecho el depósito de Ley: FA2016000064

ISSN: 2542-3371

FUNDACIÓN KOINONIA (F.K). Santa Ana de Coro, Venezuela

Ana Cristina Chirinos Martínez; Rubén Ulises Perozo Martín

virtual, bajo reglas de comunicación asíncrona. Destacamos que en este espacio, las herramientas derivadas de los cambios tecnológicos, cobran sentido, en la medida que estas sean usadas para el elevar la calidad de vida de los ciudadanos. En el contexto de la gobernabilidad y gobernanza, su uso debe apuntar a mejorar las relaciones de los gobiernos con sus ciudadanos, otras dependencias gubernamentales y con la empresa privada, a los fines de elevar los niveles de confianza de los ciudadanos en sus gobiernos, además de eliminar la opacidad en los procesos administrativos y de rendición de cuentas.

La implementación del gobierno electrónico, debe superponer los principios de calidad de los servicios, transparencia, comunicación, sobre el uso de las tecnologías de información y comunicación, preservando el derecho que tienen de estar informados, oportuna y de manera veraz, sobre los procesos de los espacios gubernamentales y la administración de los recursos del erario. En el ámbito del gobierno electrónico Venezolano, estos principios están ausentes, caracterizándose por ser portales web transaccionales, donde se colocan información, sin propiciar espacios para el intercambio de la información y muchos menos para la construcción de ideas o soluciones a los problemas de los ciudadanos. Destacamos que algunos de ellos poseen enlaces que no funcionan o están fuera de servicio, con poseen información desactualizada. Consideramos que se debe implementarse un plan rector para catapultar el gobierno electrónico, que incluya estándares de diseño y desarrollo de los portales, así como, normas de uso de los mismos.

El modelo gerencial del gobierno venezolano, es burocrático, con poco uso de las tecnologías de información, y por tanto con bajo niveles de satisfacción de los usuarios. En ese espacio de relaciones construidas a partir del uso de las herramientas de la Web 2.0, el principio rector debe ser la ética kantiana, elemento que se logra con la educación sobre las implicaciones que derivan del mal uso de ellas. De igual modo, es menester una apropiación de los conceptos de gobernabilidad y gobernanzas, por parte de los gobernantes, de manera de comprender que los servidores públicos, deben construir espacios de para la construcción de la gobernanza, involucrando a los 
Iustitia Socialis. Revista Arbitrada de Ciencias Jurídicas.

Año IV. Vol. IV. N6. Enero - Junio 2019

Hecho el depósito de Ley: FA2016000064

ISSN: 2542-3371

FUNDACIÓN KOINONIA (F.K). Santa Ana de Coro, Venezuela

Ana Cristina Chirinos Martínez; Rubén Ulises Perozo Martín

ciudadanos como actores principales en la conformación de las soluciones de los problemas.

Debe ser de preocupación de los actores gubernamentales, aplicar de manera responsable las TIC"s en sus modelos de gerencia, contemplando los niveles de accesibilidad de los ciudadanos a estas tecnologías. Partiendo del hecho que en el año 2008 con el paper White del bitcoin, nace la tecnología de las cadenas de bloques, y esto ha dado auge al internet del valor. Su uso, está revolucionando los espacios de gobiernos que apuestan por el modelo de gobierno abierto, de manera de que con su aplicación se alcanzará la transparencia gubernamental

\section{REFERENCIAS CONSULTADAS}

1. Aguilar, L. F. (2007) LA CONTRIBUCIÓN DE LA POLÍTICA PÚBLICA Y LA NUEVA GESTIÓN PÚBLICA PARA EL GOBIERNO CLAD Revista Reforma y Democracia, núm. 39, octubre, pp. 5-32 Centro Latinoamericano de Administración para el Desarrollo Caracas, Venezuela http://www.redalyc.org/pdf/3575/357533693001.pdf

2. Åke G. (2012). GOBIERNO ELECTRÓNICO - EFICIENCIA, CALIDAD DEL SERVICIO Y DEMOCRACIA. En: Åke Grönlund. Gobierno Electrónico: Diseño, Aplicaciones y Gestión. Grupo de ideas de publicación. Hershey PA.

3. Alberta. (2000). LA INICIATIVA CIBERNÉTICA. CIUDAD DE LA GRAN PRAIRIE, Alberta, Canadá. Disponible en la World Wide Web en: http://www.city.grandeprairie.ab.ca/ccy 0001.htm.

4. Armenta, Á. et al. (2012), "LA NUEVA DIVITAL DIGITAL: LA CONFLUENCIA DE LA PENETRACIÓN DE BANDA ANCHA, EL DESARROLLO SOSTENIBLE, LA ADOPCIÓN DE TECNOLOGÍA Y LA PARTICIPACIÓN COMUNITARIA", en Information Technology for Development, vol. 18, núm. 4. Londres, Reino Unido: Taylor y Francis.

5. Bertot, J.C., Jaeger, P.T., y Hansen, D. (2012). EL IMPACTO DE LAS POLÍTICAS EN EL USO DE LOS MEDIOS SOCIALES DEL GOBIERNO: problemas, desafíos y recomendaciones. Información gubernamental trimestral, 29 (1), 30-40. 
6. Brugué, Q. (2013): "UN MARCO PARA EL DESARROLLO DEL GOBIERNO ABIERTO". Revista Deliberación oㅡ 3, Dirección General de Participación Ciudadana, Acción Exterior y Cooperación del Gobierno de Aragón. Fecha de acceso: 06 de septiembre de 2018 en: http://webcache.googleusercontent.com/search?q=cache:fAsOeW7iJrsJ:aragonp articipa.aragon.es/sites/default/files/rev_deliberacion3.pdf $+\& c d=1 \& \mathrm{hl}=\mathrm{es}-419 \& \mathrm{ct}$ $=\mathrm{clnk} \& \mathrm{gl}=\mathrm{ve}$

7. Camou, A ((2001) DESAFÍOS DEL GOBIERNO DE MÉXICO: FLACSO / IISUNAM / PLAZA Y VALDÉS ESTUDIO Y COMPILACIÓN PRELIMINAR, fecha de acceso: 3 de septiembre de 2018 en: https://issuu.com/jooselitoparker/docs/los desaf os de la gobernaja

8. Carballo, Y, Cattafi, R, Sanoja A y Zambrano N. (2006). GOBIERNO ELECTRONICO EN VENEZUELA. II RT 2006-09. Técnicas de Informática ISSN 1316-6239. Fecha de acceso: 1 de septiembre de 2018 en: http://www.computacion.ciens.ucv.ve/documentos/RT-2006-09.pdf

9. Castells, M. (2001) a. LA EDAD DE LA INFORMACIÓN. LA SOCIEDAD DE RED. Vol I. Madrid.: Alianza Editorial.

10. Castells, M. (2001) b. LA GALAXIA DE INTERNET. REFLEXIONES EN INTERNET Y SOCIEDAD. Plaza \& Janés, Barcelona.

11. Deloitte. (2008). CAMBIE SU MUNDO O EL MUNDO LO CAMBIARÁ: EL FUTURO DEL GOBIERNO COLABORATIVO Y LA WEB 2.0. Obtenido de http://www.deloitte.com/view/en CA/ca/industries/19ef16bc31fb110VgnVCM1000 00ba42f00aRCRD.htm

12. EzGov FlexFoundation, (2000) "E-GOBIERNO: HACER SENTIDO UNA REVOLUCIÓN", Libro Blanco, disponible en www.ezgov.com.

13. Ferguson, M. (1999). DESARROLLOS EN GOBIERNO ELECTRONICO. EI Consejo Británico (formato pdf http://www.britishcouncil.org/governance/edigest.htm).

14.Gómez, J. (2018). EXPERIENCIA EN EL DISEÑO DE UN INSTRUMENTO DE VIRTUALIDAD. Revista Arbitrada Interdisciplinaria Koinonía, [S.I.], v. 2, n. 3, p. 65-79, feb. 2018. ISSN 2542-3088. Disponible en: http://fundacionkoinonia.com.ve/ojs/index.php/revistakoinonia/article/view/52f Fecha de acceso 06 jun. Doi 2019: http://dx.doi.org/10.35381/r.k.v2i3.52. 
Iustitia Socialis. Revista Arbitrada de Ciencias Jurídicas.

Año IV. Vol. IV. N${ }^{\circ}$. Enero - Junio 2019

Hecho el depósito de Ley: FA2016000064

ISSN: 2542-3371

FUNDACIÓN KOINONIA (F.K). Santa Ana de Coro, Venezuela

Ana Cristina Chirinos Martínez; Rubén Ulises Perozo Martín

15. González J., Pulgar A (2013). EL GOBIERNO ELECTRONICO DE VENEZUELA: NUEVOS MITOS Y REALIDADES. REVISTA DE TEORIAS, ENFOQUES Y APLICACIONES EN CIENCIAS SOCIALES. Vol. 5 No. 11 (2013). Centro occidental de la universidad "Lisandro Alvarado".

16. González, L. y Lard0one, M. (2013). FEDERALISMO Y POLÍTICA SUBNACIONAL EN PERSPECTIVA COMPARATIVA: ALGUNOS DESARROLLOS, PREGUNTAS Y PENDIENTES DE INVESTIGACIÓN POTENCIALES. Fecha de acceso: 3 de septiembre de 2018 en http://bibdigital.uccor.edu.ar/ojs/index.php/Prueba2/article/download/812/693

17. Grönlund, A. (2001) .ed. GOBIERNO ELECTRÓNICO: DISEÑO, APLICACIONES Y GESTIÓN. Hershey, PA: IDEA Group Publishing.

18. Hirst, Peter, y M. Norton (1998). "GOBIERNO ELECTRONICO- TECNOLOGÍAS DE LA INFORMACIÓN Y CIUDADANOS”. Oficina Parlamentaria de Ciencia y Tecnología, Parlamento del Reino Unido. Fecha de acceso: 3 de septiembre de 2018 en http://www.parliament.uk/post/egov.htm

19. ITA. (1999). GOBIERNO ELECTRÓNICO - UNA COLECCIÓN DE ENLACE. Instituto de Evaluación Tecnológica del proyecto Epriwatch. Disponible en la fecha de acceso a la World Wide Web: 3 de septiembre de 2018 en: http://www.oeaw.ac.at/ ita / ebene5 / e2-2a18a.htm.

20. Jaeger P.T., Bertot J.C., Shilton K. (2012). POLÍTICA DE INFORMACIÓN Y MEDIOS SOCIALES: EL GOBIERNO DE INTERCAMBIO — INTERACCIONES DE CIUDADANAS WEB 2.0. En: Reddick C., Aikins S. (eds) Tecnologías Web 2.0 y gobernabilidad democrática. Administración pública y tecnología de la información, vol 1. Springer, Nueva York, NY

21. Kemp, S. (2018). DIGITAL EN 2018. PUNTOS ESENCIALES EN INTERNET, MEDIOS SOCIALES, MÓVILES Y ECOMERIOS UTILIZADOS EN TODO EL MUNDO. Fecha de acceso: 3 de septiembre de 2018 en https://wearesocial.com/blog/2018/01/global-digital-report-2018.

22. Lorenzi, David y otros (2014), "MEJORANDO LA EXPERIENCIA DEL SERVICIO GUBERNAMENTAL A TRAVÉS DE QRCODES EN PLATAFORMAS MÓVILES", en Government Information Quarterly, vol. 31, núm.1, Amsterdam, Holanda: Elsevier.

23. Olnesa S., Ubachtb J., Janssen M. BLOQUEO EN EL GOBIERNO: BENEFICIOS E IMPLICACIONES DE LA TECNOLOGÍA LEDGER DISTRIBUIDA PARA EL 
Iustitia Socialis. Revista Arbitrada de Ciencias Jurídicas.

Año IV. Vol. IV. N${ }^{\circ}$. Enero - Junio 2019

Hecho el depósito de Ley: FA2016000064

ISSN: 2542-3371

FUNDACIÓN KOINONIA (F.K). Santa Ana de Coro, Venezuela

Ana Cristina Chirinos Martínez; Rubén Ulises Perozo Martín

INTERCAMBIO DE INFORMACIÓN. Información gubernamental Trimestral 34 (2017) 355-364.

24. Oszlak, O. (2012). GOBIERNO ABIERTO: PROMESAS Y DESAFÍOS. Buenos Aires: Cedes. Plan Fénix. (P.68-p.75) fecha de acceso: 03 de Septiembre de 2018 en: http://www.vocesenelfenix.com/sites/default/files/pdf/Oszlak.pdf

25. Pardo, T (2000) REALIZANDO LA PROMESA DEL GOBIERNO DIGITAL: ES SU MORETANO CONSTRUYENDO UN SITIO WEB. IMP Mag Disponible en http://www.cisp.org/imp/october2000/1000pardo.htm

26. Reddick C. y Aikins S (2012). TECNOLOGÍAS WEB 2.0 Y GOBIERNO DEMOCRÁTICO. ADMINISTRACIÓN PÚBLICA Y TECNOLOGÍA DE LA INFORMACIÓN, vol 1. Springer, Nueva York, NY

27. Sanz J. (2000). ¿QUÉ ES LA SOCIEDAD DEL CONOCIMIENTO? Nueva revista de política, cultura y arte. Fecha de acceso: 06 de septiembre de 2018 en: https://www.nuevarevista.net/revista-sociedad/que-es-la-sociedad-delconocimiento/

28. Tapscott, D. (2010). BIBLIOGUIAS-BIBLIOTECA CENTRAL. Consultado el 6 de septiembre de 2018 en: http://biblioguias.cepal.org/c.php?g=159545\&p=1044390

29. Naciones Unidas. ENCUESTA DE GOBIERNO ELECTRÓNICO 2018. GESTIÓN DE GOBIERNO ELECTRÓNICO PARA APOYAR LA TRANSFORMACIÓN HACIA SOCIEDADES SOSTENIBLES Y RESILIENTES. Fecha de acceso: 8 de septiembre de 2018 en https://www.unescap.org/resources/e-governmentsurvey-2018-gearing-e-government-support-transformation-towards-sustainable.

30. Van Dijck, J. (2016). LA CULTURA DE LA CONECTIVIDAD: UNA HISTORIA CRÍTICA DE LAS REDES SOCIALES. Buenos Aires: Siglo XXI. Ediciones del siglo XXI. Fecha de acceso 1 de septiembre de 2018 en: http://pdfhumanidades.com/sites/default/files/apuntes/La-cultura-de-laconectividad_-Jose-Van-Dijck.pdf

31. Webb P.L. (2012). MICROBLOGACIÓN: UN ANÁLISIS DE LAS POLÍTICAS DE EMISIÓN DEL GOBIERNO Y LAS MEJORES PRÁCTICAS. EN: REDDICK C., AIKINS S. (EDS) TECNOLOGÍAS WEB 2.0 Y GOBIERNO DEMOCRÁTICO. ADMINISTRACIÓN PÚBLICA Y TECNOLOGÍA DE LA INFORMACIÓN, vol 1. Springer, Nueva York, NY

32. WEFFER, Nataly María. EL USO DE LAS NOTIFICACIONES POR CORREO ELECTRÓNICO EN EL SISTEMA JUDICIAL Y SU FUNCIÓN EN LA 
Iustitia Socialis. Revista Arbitrada de Ciencias Jurídicas.

Año IV. Vol. IV. N${ }^{\circ}$. Enero - Junio 2019

Hecho el depósito de Ley: FA2016000064

ISSN: 2542-3371

FUNDACIÓN KOINONIA (F.K). Santa Ana de Coro, Venezuela

Ana Cristina Chirinos Martínez; Rubén Ulises Perozo Martín

ADMINISTRACIÓN DE JUSTICIA VENEZOLANA. IUSTITIA SOCIALIS, [S.I.], v. 2, n. 3, p. 38-53, marzo de 2018. ISSN 2542-3371. Fecha de acceso: 23 de febrero de 2019 Disponible en: http://fundacionkoinonia.com.ve/ojs/index.php/lustitia_Socialis/article/view/93/78

\section{REFERENCES CONSULTED}

1. Aguilar, L. F. (2007) THE CONTRIBUTION OF PUBLIC POLICY AND THE NEW PUBLIC MANAGEMENT TO GOVERNANCE CLAD Magazine Reforma y Democracia, núm. 39, October,, pp. 5-32 Latin American Center for Administration for Development Caracas, Venezuela http://www.redalyc.org/pdf/3575/357533693001.pdf

2. Åke G. (2012). ELECTRONIC GOVERNMENT-EFFICIENCY, SERVICE QUALITY AND DEMOCRACY. In: Åke Grönlund. Electronic Government: Design, Applications and Management. Idea Group Publishing. Hershey PA.

3. Alberta. (2000). THE CYBERCITY INITIATIVE. CITY OF THE GRAND PRAIRIE, Alberta, Canada. Available on the World Wide Web at: http://www.city.grandeprairie.ab.ca/ccy 0001.htm.

4. Armenta, Á. et al. (2012), "THE NEW DIGITAL DIVIDE: THE CONFLUENCE OF BROADBAND PENETRATION, SUSTAINABLE DEVELOPMENT, TECHNOLOGY ADOPTION AND COMMUNITY PARTICIPATION", in Information Technology for Development, vol. 18, núm. 4. London, United Kingdom: Taylor y Francis.

5. Bertot, J.C., Jaeger, P.T., \& Hansen, D. (2012). THE IMPACT OF POLICIES ON GOVERNMENT SOCIAL MEDIA USAGE: Issues, challenges, and recommendations. Government Information Quarterly, 29(1), 30-40.

6. Brugué, Q. (2013): "A FRAMEWORK FOR DEVELOPING OPEN GOVERNMENT". Deliberación Magazine no 3, General Directorate of Citizen Participation, External Action and Cooperation of the Government of Aragon. Access date: September 06, 2018 in: http://webcache.googleusercontent.com/search?q=cache:fAsOeW7iJrsJ:aragonparticipa.ar agon.es/sites/default/files/rev deliberacion3.pdf+\&cd=1\&hl=es-419\&ct=clnk\&gl=ve

7. Camou, A ((2001) CHALLENGES OF GOVERNANCE MEXICO: FLACSO / IISUNAM / PLAZA Y VALDÉS PRELIMINARY STUDY AND COMPILATION, access date: September 3, 2018 at: https://issuu.com/jooselitoparker/docs/los desaf os de la gobernabilidad 
Iustitia Socialis. Revista Arbitrada de Ciencias Jurídicas.

Año IV. Vol. IV. N6. Enero - Junio 2019

Hecho el depósito de Ley: FA2016000064

ISSN: 2542-3371

FUNDACIÓN KOINONIA (F.K). Santa Ana de Coro, Venezuela

Ana Cristina Chirinos Martínez; Rubén Ulises Perozo Martín

8. Carballo, Y, Cattafi, R, Sanoja A and Zambrano N. (2006). ELECTRONIC GOVERNMENT IN VENEZUELA. II RT 2006-09.Lectures in Computer Science ISSN 1316-6239. Access date: September 01, 2018 at: http://www.computacion.ciens.ucv.ve/documentos/RT-2006-09.pdf

9. Castells, M. (2001)a. THE AGE OF INFORMATION. THE NETWORK SOCIETY. Vol I. Madrid .: Editorial Alliance.

10. Castells, M. (2001)b. THE GALAXY INTERNET. REFLECTIONS ON THE INTERNET AND SOCIETY. Plaza \& Janés, Barcelona.

11. Deloitte. (2008). CHANGE YOUR WORLD OR THE WORLD WILL CHANGE YOU: THE FUTURE OF COLLABORATIVE GOVERNMENT AND WEB 2.0. Retrieved from http://www.deloitte.com/view/en CA/ca/industries/19ef16bc31fb110VgnVCM100000 ba42f00aRCRD.htm

12. EzGov FlexFoundation, (2000) 'E-GOVERNMENT: MAKING SENSE OF A REVOLUTION', White paper, available at www.ezgov.com.

13. Ferguson, M. (1999). DEVELOPMENTS IN ELECTRONIC GOVERNANCE. The British Council (pdf-format http://www.britishcouncil.org/governance/edigest.htm).

14. Gómez, J. (2018). EXPERIENCE IN THE DESIGN OF AN INSTRUMENT FOR VIRTUALITY. Revista Arbitrada Interdisciplinaria Koinonía, [S.I.], v. 2, n. 3, p. 6579, feb. 2018. ISSN 2542-3088. Available at: http://fundacionkoinonia.com.ve/ojs/index.php/revistakoinonia/article/view/52f Access date 06 jun. 2019 doi: http://dx.doi.org/10.35381/r.k.v2i3.52.

15. González J., Pulgar A (2013). THE ELECTRONIC GOVERNMENT IN VENEZUELA: NEW MYTHS AND REALITIES. MAGAZINE THEORIES, APPROACHES AND APPLICATIONS IN SOCIAL SCIENCES. Vol. 5 No. 11 (2013). Western Center University "Lisandro Alvarado".

16. González, L. and Lard0one, M. (2013). FEDERALISM AND SUBNATIONAL POLITICS IN COMPARATIVE PERSPECTIVE: SOME DEVELOPMENTS, QUESTIONS AND POTENTIAL RESEARCH AGENDS. Access date: September 03, 2018 at http://bibdigital.uccor.edu.ar/ojs/index.php/Prueba2/article/download/812/693

17. Grönlund, A. (2001) .ed. ELECTRONIC GOVERNMENT: DESIGN, APPLICATIONS, AND MANAGEMENT. Hershey, PA: IDEA Group Publishing. 
18. Hirst, Peter, y M. Norton (1998). "ELECTRONIC GOVERNMENT- INFORMATION TECHNOLOGIES AND CITIZEN". Parliamentary Office of Science and Technology, United Kingdom Parliament. Access date: September 03, 2018 at http://www.parliament.uk/post/egov.htm

19. ITA. (1999). ELECTRONIC GOVERNMENT--A LINK COLLECTION. Institute of Technology Assessment Epriwatch project. Available on the World Wide Web Access date: September 03, 2018 at at: http://www.oeaw.ac.at/ ita/ebene5/e22a18a.htm.

20. Jaeger P.T., Bertot J.C., Shilton K. (2012). INFORMATION POLICY AND SOCIAL MEDIA: FRAMING GOVERNMENT-CITIZEN WEB 2.0 INTERACTIONS. In: Reddick C., Aikins S. (eds) Web 2.0 Technologies and Democratic Governance. Public Administration and Information Technology, vol 1. Springer, New York, NY

21. Kemp, S. (2018). DIGITAL IN 2018. ESSENTIALS INSIGHTS INTO INTERNET, SOCIAL MEDIA, MOBILE, AND ECOMERCE USED AROUND THE WORLD. Access date: September 03, 2018 at https://wearesocial.com/blog/2018/01/globaldigital-report-2018.

22. Lorenzi, David et al.(2014), "ENHANCING THE GOVERNMENT SERVICE EXPERIENCE THROUGH QRCODES ON MOBILE PLATFORMS", en Government Information Quarterly, vol. 31, núm.1, Amsterdam, Holanda: Elsevier.

23. Olnesa S., Ubachtb J., Janssen M. BLOCKCHAIN IN GOVERNMENT: BENEFITS AND IMPLICATIONS OF DISTRIBUTED LEDGER TECHNOLOGY FOR INFORMATION SHARING. Government Information Quarterly 34 (2017) 355-364.

24. Oszlak, O. (2012). GOBIERNO ABIERTO: PROMESAS Y DESAFÍOS. Buenos Aires: Cedes. Plan Fénix.(p.68-p.75) fecha de acceso: 03 de Septiembre de 2018 en: http://www.vocesenelfenix.com/sites/default/files/pdf/Oszlak.pdf

25. Pardo, T (2000) REALZING THE PROMISE OF DIGITAL GOVERNMENT: IT"S MORETHAN BUILDING A WEB SITE. IMP Mag Disponible en http://www.cisp.org/imp/october2000/1000pardo.htm

26. Reddick C. y Aikins S (2012). WEB 2.0 TECHNOLOGIES AND DEMOCRATIC GOVERNANCE. PUBLIC ADMINISTRATION AND INFORMATION TECHNOLOGY, vol 1. Springer, New York, NY

27. Sanz J. (2000). WHAT IS THE KNOWLEDGE SOCIETY? New Journal of Politics, Culture and Art. Access date: September 06, 2018 in: https://www.nuevarevista.net/revista-sociedad/que-es-la-sociedad-del-conocimiento/ 
28. Tapscott, D. (2010). BIBLIOGUIAS-CENTRAL LIBRARY. Retrieved on September 6, 2018 at: http://biblioguias.cepal.org/c.php?g=159545\&p=1044390

29. United Nations. E-GOVERNMENT SURVEY 2018. GEARING E-GOVERNMENT TO SUPPORT TRANSFORMATION TOWARDS SUSTAINABLE AND RESILIENT SOCIETIES. Access date: September 08, 2018 at https://www.unescap.org/resources/e-government-survey-2018-gearing-egovernment-support-transformation-towards-sustainable.

30. Van Dijck, J. (2016). THE CULTURE OF CONNECTIVITY: A CRITICAL HISTORY OF SOCIAL NETWORKS. Buenos Aires: Siglo XXI. Editions 21st century. Access date September 1, 2018 in: http://pdfhumanidades.com/sites/default/files/apuntes/La-cultura-de-laconectividad_-Jose-Van-Dijck.pdf

31. Webb P.L. (2012). MICROBLOGGING: AN ANALYSIS OF GOVERNMENT ISSUED POLICIES AND BEST PRACTICES. IN: REDDICK C., AIKINS S. (EDS) WEB 2.0 TECHNOLOGIES AND DEMOCRATIC GOVERNANCE. PUBLIC ADMINISTRATION AND INFORMATION TECHNOLOGY, vol 1. Springer, New York, NY

32. WEFFER, Nataly María. THE USE OF THE NOTIFICATIONS BY ELECTRONIC MAIL IN THE JUDICIAL SYSTEM AND ITS ROLE IN THE VENEZUELAN JUSTICE ADMINISTRATION. IUSTITIA SOCIALIS, [S.I.], v. 2, n. 3, p. 38-53, Mar 2018. ISSN 2542-3371. Access date: 23 Feb. 2019 Available at: http://fundacionkoinonia.com.ve/ojs/index.php/lustitia Socialis/article/view/93/78

2019 por los autores. Este artículo es de acceso abierto y distribuido según los términos y condiciones de la licencia Creative Commons Atribución-NoComercial-Compartirlgual 4.0 Internacional (CC BY-NC-

SA 4.0) (https://creativecommons.org/licenses/by-nc-sa/4.0/). 